\title{
Assessment procedures in anonymously written threats of harm and violence
}

\author{
Isabelle van der Vegt ${ }^{1}$, Pippa Gregory ${ }^{2}$, Bram B. van der Meer ${ }^{3}$, Junyi Yang ${ }^{4}$, Bennett Kleinberg ${ }^{1,5}$, \\ and Paul Gill ${ }^{1}$ \\ ${ }^{1}$ Department of Security and Crime Science, University College London \\ ${ }^{2}$ Major Crime Investigative Support, National Crime Agency, United Kingdom \\ ${ }^{3}$ Dantes Psychology Services, The Netherlands \\ ${ }^{4}$ Institute of Criminology, Cambridge University \\ ${ }^{5}$ Department of Methodology and Statistics, Tilburg University \\ Correspondence: isabelle.vandervegt@ucl.ac.uk
}

This project has received funding from the European Research Council (ERC) under the European

Union's Horizon 2020 research and innovation programme (Grant Agreement No. 758834).

\begin{abstract}
Anonymously written threats constitute a special form of worrying behavior, in which the author of a threat decides to hide their identity. Importantly, anonymous threats are an increasingly common issue exacerbated by online communication. Anonymity raises additional challenges for threat assessors, but little is known about how practitioners approach such cases. Although a large body of research exists concerning assessment procedures of nonanonymous cases, little is known about how experts approach a case based merely on communications and in the absence of detailed contextual information. Therefore, the current study serves to gain a better picture of the assessment procedures associated with anonymous threatening communications (ATCs). Extensive semi-structured interviews were conducted with expert threat assessors, in which participants were asked about their general approach to ATCs, as well as their thoughts regarding a real-life case. Responses were qualitatively coded, in order to reveal common themes and divergent approaches. Based on the results obtained, we formulate eight recommendations for the furtherance of the field of anonymous threat assessment.
\end{abstract}

\section{Public Significance Statement}

This study examined assessment procedures in the case of anonymous threatening communications by interviewing threat assessment experts on their approach to the same case. Experts largely agreed on their assessment of violence risk, but were highly divergent in their management advice, assessments of communication content, and suspected characteristics of the author. We suggest that the assessment of anonymous threatening communications will benefit from further structuring, and provide suggestions for doing so.

Keywords: anonymous threatening communication, threat assessment, worrying communication, written threats

Supplemental materials: https://doi.org/10.1037/tam0000168.supp 
(C)American Psychological Association, 2022. This paper is not the copy of record and may not exactly replicate the authoritative document published in the APA journal. The final article is available, upon publication, at: https://doi.org/10.1037/tam0000168

Anonymous threats span a wide range of contexts, from private individuals receiving letters with peculiar content, to worrying communications directed at public figures such as celebrities, government officials or well-known business executives, and contamination threats to businesses or bomb threats sent to public places (Calhoun \& Weston, 2017; Simons \& Tunkel, 2013, 2021). The assessment and management of such written communication form an important part of the work of threat assessment experts. People often experience significant fear when receiving unwanted letters or messages, and also struggle with several questions when falling victim to such acts. Questions range from 'how dangerous is this person', 'will this escalate to serious violence' to 'what can I do to make this stop' and 'should I take measures to protect myself and my family'. It should be within the range of the expertise of a threat assessment professional to address these questions, manage the victim's fear and take necessary steps when protection is needed.

When the author of a threat decides to hide his/her identity, this may pose additional challenges for threat assessors. For example, anonymous threats raise questions about the expected characteristics of the unknown offender, what the individual's motive is to remain anonymous, and what the anonymity means in relation to the seriousness of the threat and associated danger (Simons \& Tunkel, 2021). Moreover, case experience indicates that anonymity often increases the victim's fear, particularly when multiple communications are received. This is most likely related to the extra uncertainty that the anonymity adds to the victim's already existing apprehension.

A significant body of research concerning threats and assessment procedures in the case of a known source exists (e.g., Borum et al., 1999; Calhoun \& Weston, 2017; Meloy \& Hoffmann, 2013), but little has been written about anonymous threats and how to conduct assessments in such cases (Simons \& Tunkel, 2021). One possible explanation for this is that threatening communications (studied) in the past were often not anonymous. In a 1991 study of threatening letters to US Congress members, $81 \%$ of writers gave their full name, $74 \%$ gave an address, and $86 \%$ gave some (other form of) identifying information, whereas just $14 \%$ of letter writers remained anonymous (Dietz et al., 1991). Similar patterns were observed in letters directed at celebrities $(N=214)$, with just $5 \%$ of writers maintaining anonymity and $95 \%$ giving some form of identifying information in their (first) communication (Dietz et al., 1991). Although threat authors may continue to identify themselves in some cases, the internet has significantly facilitated the process of anonymously sending threatening communications. Simons \& Tunkel (2013) predicted that anonymous threats would become more common and time-consuming, noting at the time that the majority of threats handled by the FBI's Behavioral Analysis Unit were anonymous in nature. Although the (previously) low rate of anonymous threatening communications may explain the lack of research in this area thus far, we acknowledge that this is an increasingly common issue exacerbated by online communication, and thus warrants further attention (Simons \& Tunkel, 2013, 2021; Wallace, 2015).

When assessing a threat from a known source, threat assessors will tend to focus less on the communication itself, its content, physical traces, and other forensic evidence, but instead will focus on the author's personal characteristics: gathering and evaluating personal, historical, contextual, and clinical information about the threatener (Monahan et al., 2001). In the case of an anonymous threat, such information is obviously lacking. Instead, characteristics, motivation and intent of the author have to be uncovered and interpreted from the communication. An anonymous threatener may also include misleading information in order to further conceal intent or identity (e.g., an individual posing as a group of threateners). Moreover, in the case of a known threatener, practitioners can turn to several violence risk assessment tools deriving from the Structured Professional Judgment (SPJ) model (see e.g., 
(C)American Psychological Association, 2022. This paper is not the copy of record and may not exactly replicate the authoritative document published in the APA journal. The final article is available, upon publication, at: https://doi.org/10.1037/tam0000168

Hart et al., 2016). In contrast, there is no reference in the academic literature to validated tools which can help the analyst to structure the assessment in the specific case of an anonymous threat. A major reason for conducting this study and continuing research in this specific field is that to this day, no standardized protocol for assessing anonymous communicated threats exists. In order to arrive at such a method, first more needs to be known about how threat assessment professionals and related professionals such as police officers, psychologists or security specialists attempt to analyze anonymous cases. With this paper, we aim to shed light on these procedures. Before doing so, we briefly review the body of literature that has previously discussed approaches to anonymous threats.

\section{Previous work on anonymous threat assessment}

Simons \& Tunkel $(2013,2021)$ described the procedure of assessing anonymous threats adhered to by the North-American Federal Bureau of Investigation's (FBI) Behavioral Analysis Unit (BAU). It is emphasized that a team of evaluators is needed to carry out this process. The BAU will also designate a lone assessor who assesses the ATC in isolation from the rest of the team in order to manage confirmation bias. Furthermore, the lead member of the team will gather and distribute information on the threat. This includes seeking to answer triage questions relating to the delivery of the threat, the feasibility of the threat, a possible relationship between the victim and offender, the characteristics of the target, and other contextual information (Simons \& Tunkel, 2021). In contrast, the lone assessor focuses on possible linguistic staging (e.g., the use of plural pronouns by a possible single author), possible motives, cues to deception (e.g., level of detail in the threat), as well as the resolution to violence and its possible imminence. Although the lone assessor also has access to information on the ATC's mode of delivery, they are not provided with further contextual information regarding the target, again in an attempt to limit confirmation bias. After the threat is independently reviewed by the other team members, they will reconvene to reach a group assessment. Thereafter, the lone assessor presents their assessment to the team which was achieved without access to background and victim information. The full team will then reevaluate their conclusions in light of the new assessment. The final resulting assessment typically discusses threat-enhancing and mitigating factors, as well as a recommended threat management strategy (Simons \& Tunkel, 2013, 2021). Although the described procedure using a lone assessor may not be applicable or possible in all circumstances, the authors importantly state that threat assessment teams need to have an established, consistent procedure in place for the effective management of anonymous threats (Simons \& Tunkel, 2021).

A notable example of an SPJ tool applicable to ATCs is The Communications Threat Assessment Protocol-25 (CTAP-25), which has been specifically developed for the assessment of problematic communications (James et al., 2014). The CTAP-25 was not developed for anonymous cases specifically, but due to its focus on risk factors that can be derived from the communication itself, it may be of particular relevance to anonymous cases where a threat manager does not have much information besides the communication. Although the developers state that the instrument makes use of empirically defined risk factors, an evaluation has yet to be published (Geurts et al., 2017).

One aspect specifically relevant when dealing with anonymous communications is the field of forensic linguistics, often referred to as 'linguistic profiling', suggesting that aspects like the author's choice of words, spelling and grammar can provide insight into the offender's personal characteristics. In a survey of threat assessment professionals, experts were asked which linguistic factors they attend to in order to assess the risk of violence (van der Vegt, Mozes, et al., 2020). Again, this is particularly relevant to anonymous cases, where the 
(C)American Psychological Association, 2022. This paper is not the copy of record and may not exactly replicate the authoritative document published in the APA journal. The final article is available, upon publication, at: https://doi.org/10.1037/tam0000168

language in a communication is sometimes the only available evidence. A total of 21 participants with an average of 16 years of threat assessment experience disclosed what they look for in a text to assess its threat level. The free text responses covered a wide variety of factors. Several variables related to the content of the communication itself, for example whether the author made a direct threat or whether they spoke of violence, demonstrated paranoia, or a romantic relationship. Participants also noted that the emotional tone of the communication would be of interest, such as anger or frustration. Several other cues related to mental health, such as possible signs of psychosis, delusional jealousy, or suicidal ideation in the language use. Lastly, experts noted they take into account unusual grammar, incoherence, as well as handwriting and the inclusion of drawings or images. The psycholinguistic factors raised by survey participants were used to create the Grievance Dictionary, a tool aimed at automatically analyzing threatening language use, which measures concepts such as planning, violence, paranoia, and weaponry in texts (van der Vegt, Mozes, et al., 2020).

All in all, even though a procedure in assessing ATCs has been documented (Simons $\&$ Tunkel, 2013, 2021) and some tools have emerged that are specifically aimed at assessing communications, no single standardized procedure, guideline or Structural Professional Judgment tool exists for anonymous communications. This lack of structure and consistency has the potential to open practitioners up to criticism as the process becomes reliant on subjective and intuitive interpretation. It raises the possibility of important violence risk factors being missed or overlooked in ATCs, or assessors being particularly sensitive to bias while conducting assessments. Whilst biases in themselves do not exclusively equate to errors in decision-making, they are generally regarded as systematic, suboptimal judgment ${ }^{1}$ (Keren \& Teigen, 2004). Unconscious bias in particular, to which we are all prone, renders the threat assessor vulnerable to making unreliable inferences unless techniques are incorporated into our practice to mitigate against them. The first step to managing bias is, undoubtedly, an acknowledgement that they exist and an understanding of how they operate, which is discussed in the next section.

\section{The impact of bias on threat assessment procedures in anonymous cases}

An increasing amount of research on threat assessment focusses on the procedure itself. In particular, researchers and practitioners have become aware of various biases associated with determining the risk of violence, such as confirmation bias (Nickerson, 1998), selective perception (Murray \& Thomson, 2010) and illusory correlations (Chapman \& Chapman, 1967). The risk of biased decision making may be particularly high in the case of ATCs, due to the limited information available, as well as the lack of structure and standardized protocols in assessment procedures.

Confirmation bias involves the 'seeking or interpreting of evidence in ways that are partial to existing beliefs, expectations, or a hypothesis in hand' (Nickerson, 1998). The danger of this arising in threat assessment has been raised on several occasions (Meloy, 2015; Simons \& Tunkel, 2013, 2021). The concept is closely related to selective perception, where individuals specifically attend to information which confirms their expectations and thus influences their judgment (Murray \& Thomson, 2010). This process may be particularly exacerbated by the limited amount of information available in ATCs. For example, if a suspect is targeting a male victim with repeated romantic and obsessive communications, the

\footnotetext{
${ }^{1}$ It must however be noted that another school of thought (see e.g., Gigerenzer, 2008) views biases as useful heuristics that can aid in processing complex information and - in some situations - lead to more accurate decisions. Nevertheless, it remains important to examine the effect of these phenomena on (anonymous) threat assessment.
} 
practitioner may assume the suspect is female and as a result may particularly attend to cues which suggest this is the case. They may pay particular attention to gendered (female) language in the communication or attribute the handwriting to a woman, confirming their preconceived notion and disregarding the possibility that the suspect could be male instead.

Anchoring bias is when an individual strongly relies on an initial piece of salient information (Iverson et al., 2008; Tversky \& Kahneman, 1974). This information then disproportionally affects the judgment of said individual. In threat assessment, this may occur when an assessor is briefed on a case by someone who is highly concerned about the risk of violence or when very violent threats are made at the beginning of a communication, affecting subsequent assessments.

Illusory correlations influence judgment when a practitioner believes there to be a correlation between concepts that do not exist in practice (Chapman \& Chapman, 1967). The danger of erroneously correlating risk cues to risk of violence has received some attention in the field of threat assessment and violence risk assessment (Elbogen, 2002; Murray \& Thomson, 2010). With limited research on violence risk cues in the specific case of anonymous communications, particularly when it comes to linguistic cues, practitioners may particularly rely on illusory correlations, possibly brought about by previous experience. For example, if a practitioner previously found that someone who stressed the imminence of violence in their communication indeed quickly actualized their threat, they may falsely assume this to be a violence risk factor in future. This then disregards the possibility of someone who merely wants to cause fear and thus stresses imminence, but who never plans to commit a violent act.

In addition, threat managers may also base their assessment on illusory correlations between communication content and the profile of a threat author. For example, if a practitioner previously experienced that someone who wrote incoherently was disordered, they may expect this to be true in future cases as well, disregarding the possibility that a threat author may write incoherently on purpose. Similar problems may arise if a practitioner has particular beliefs about the language use associated with gender or specific age groups. All in all, several biases may arise in the process of threat assessment, and little is known about how these manifest in the case of anonymous threatening communications.

\section{The current study}

The present research serves to gain a better picture of the assessment procedures experts follow in the case of anonymous threatening communications. Research on assessment procedures is prevalent in the case of non-anonymous cases, but little is known about how experts approach a case based merely on communications and in the absence of detailed contextual information.

Using an extensive semi-structured interview, expert practitioners were asked about their general approach to ATCs, as well as their thoughts regarding a specific real-life case. Responses were qualitatively coded, in order to reveal common themes and divergent approaches. Based on the results obtained, we formulate recommendations for the furtherance of the field of anonymous threat assessment.

\section{Method}

\section{Participants}

Threat assessment experts were approached through the professional contacts of the paper's authors, some of which conduct research on threat assessment or are threat assessment professionals with several years of experience in the public and private sector. Interviewees were further approached at the 2019 Association for European Threat Assessment 
(C)American Psychological Association, 2022. This paper is not the copy of record and may not exactly replicate the authoritative document published in the APA journal. The final article is available, upon publication, at: https://doi.org/10.1037/tam0000168

Professionals conference (Rotterdam, The Netherlands), as well as the 2019 Grievances and Grudges conference (Cambridge, United Kingdom). The majority of interviews were conducted at the conferences, or shortly after via video calls. Through snowball sampling further participants were identified, resulting in a total sample of $N=13$ interviewees.

\section{Procedure}

The study procedure was approved by the departmental ethics committee. After participants read the study information sheet and signed a consent form, the interviews were started. The semi-structured interviews took approximately one hour to complete (see online supplemental materials ${ }^{2}$ for all materials). Participants were first asked about their background within the field of threat assessment and their experience with anonymous threats in particular. They then read two (copies of) anonymous threat letters. Both letters were handwritten on paper and contained 134 and 159 words, respectively. Identifying information of the case (names of persons or places) were redacted. The participants were provided with some contextual information, namely that the first letter was sent to a supermarket branch and the second to a local church approximately two months later. Although both letters were presented as part of the case, participants were not informed whether the two letters were connected. They only knew that the hypothetical security manager of the supermarket who contacted them to assess the case was in possession of both letters. That is, the supermarket and church were in the vicinity of each other and both received worrying communications. In the first letter, the author threatened to contaminate products in the supermarket and subsequently demanded a large amount of cash in order to evade police. In the second letter, the author threatened to torture a child and bomb the church, in addition to demanding the church stop its services. The interviewers were not aware of the outcome of the case or of any contextual information beyond that provided to the interviewees.

It must be noted that the letters used in this paper are drawn from a real-life case. However, the suspect was apprehended before they could engage in any act of violence. This is the main reasons why we were able to make use of the letters in this study.

Participants had up to twenty minutes to read the communications, but most did not take the full amount of time. They were then asked about various aspects of the case, including their thoughts on possible characteristics of the author, the level of risk associated with the case, and appropriate security measures. Finally, interviewees were asked about their confidence in assessing the case.

\section{Analysis}

All interviews were transcribed and subsequently analyzed for recurring themes using NVivo software (QSR Internation Pty Ltd., 2014), which can be used to qualitatively organize and analyze interview data. Each interview question was considered as a separate node, and responses categorized according to themes. For example, the node 'professional background' may contain themes such as 'psychology', 'psychiatry', and 'police', with sections of interview transcripts highlighted for the theme they belong to (e.g., 'I attended the police academy' would be coded as part of the theme 'police'). Consequently, percentages can be calculated for each theme (i.e., the proportion of interviewees who mentioned the theme). Note that percentages for each theme do not always add up to $100 \%$ because a single participant may have mentioned several themes in their response. For example, a participant may have obtained a degree in

\footnotetext{
${ }^{2}$ https://osf.io/5twzu/?view_only=0c7744e97bd74957ac30f06e44337c7b
} 
psychology and attended the police academy. All percentages reported in the results were derived from this qualitative coding procedure in NVivo.

\section{Results}

Background of sample

On average, the thirteen participants had 18.10 years of experience with threat assessment, ranging between 6 months to 30 years. A large number of interviewees worked for or owned a private threat assessment company (46.15\%). Others worked for the police $(30.77 \%)$, government $(7.69 \%)$, mental health services $(7.69 \%)$, or a university $(7.69 \%)$. Their work concerns a wide variety of cases, such as threats to public figures, threats to schools and universities, workplace violence, and counter-terrorism.

The majority of the participants had a background in psychology $(84.62 \%)$, with two participants having a psychiatry degree (15.38\%) and some (additionally) attended a police or military academy (23.08\%). None of the participants mentioned specific formal training (e.g., a degree or course) in threat assessment. Participants mentioned they learned through experience, mentorship and attending conferences:

"I joined the Association for Threat Assessment Professionals in [YEAR] and started going to those meetings, presenting there. And that's really my training. And going to courses, going to people's lectures, teaching it."

"And the model of learning how to do threat assessment, still today, but definitely back then, it was brand new as an art and science. So, it was taught through mentorship."

Interviewees generally reported to have worked many anonymous threat cases $(46.15 \%)$ ranging between a dozen to 100 a year. Some were unsure how many $(7.69 \%)$ or reported to work just a few (30.77\%):

"Very few, very few [anonymous cases]. Just the vast majority of the cases are being signed because people want to make their point. [...] The vast majority, they just sign and give their details and where they live, and telephone number and email accounts."

The majority of interviewees stated they were confident when dealing with anonymous cases in general $(53.85 \%)$. Others were moderately confident $(7.69 \%)$, not confident $(7.69 \%)$ or unsure about their confidence level (7.69\%). On the topic of confidence, one participant added:

"I do not work on it myself so the confidence derives from the fact that I work in a team. So, it's like a team confidence - I'm very confident in the team. And in the ability of the team to change their hypothesis."

Interviewees were also asked when they may not want to be involved in assessing an anonymous case, with responses shown in Table 1. 
CAmerican Psychological Association, 2022. This paper is not the copy of record and may not exactly replicate the authoritative document published in the APA journal. The final article is available, upon publication, at: https://doi.org/10.1037/tam0000168

Table 1

Themes regarding appropriateness of involvement with a case

\begin{tabular}{lll}
\hline When is involvement inappropriate? & \% & $\boldsymbol{n}$ \\
\hline If professional has no expertise on subject matter & 38.46 & 5 \\
Involvement is always appropriate & 15.38 & 2 \\
If there is little context information available & 15.38 & 2 \\
If it is a low risk case & 7.69 & 1 \\
If target is uncooperative & 7.69 & 1 \\
If required to work alone & 7.69 & 1 \\
\hline
\end{tabular}

\section{General approach to anonymous threats}

When asked about the general steps taken in assessing anonymous communications, several approaches were mentioned. The majority of interviewees mentioned they would want to seek further information besides the communication (76.92\%):

"I would ask for any other information that they have. Did they have any other types of communications. Any other strange incident that they might worry about in the last couple of months."

"I want to have information on possible suspicious activities around the victim or not, and I want to know about possible conflicts. I want to know more about the victim itself."

Several interviewees also mentioned they would examine the content of the threat $(46.15 \%)$; for example, to look for aggressive or threatening language. Some participants also mentioned they would consult other experts $(61.54 \%)$ such as a linguist or a private investigator. Some participants mentioned they make use of a professional judgment tool, framework, or software (38.46\%) such as the CTAP-25, the JACA principle ${ }^{3}$ or threattriage.com (a resource for automatic linguistic analysis, see: Smith et al., 2013), respectively.

\section{Assessment of the specific case}

\section{First thoughts}

After reading the two letters, the interviewees were asked to report their first thoughts on the case. The majority of interviewees made remarks about the linguistic content and style in the communications $(92.31 \%)$. In the study of language use, linguistic content generally refers to the language use under the conscious control of the author (e.g., use of specific nouns and verbs) whereas linguistic style is assumed to not be under one's conscious control (e.g., use of pronouns and spelling mistakes; see e.g. Goswami et al., 2009; Holmes, 1998; Pennebaker, 2011a, 2011b). Other interviewees also mentioned the handwriting in the communications $(30.77 \%)$ :

"There's a spelling mistake there, there's spelling mistakes here as well. Also, there is similarity in use of language, although this one is more

\footnotetext{
${ }^{3}$ Searching for Justification, Alternatives, Consequences and Ability (de Becker, 1998)
} 
(C)American Psychological Association, 2022. This paper is not the copy of record and may not exactly replicate the authoritative document published in the APA journal. The final article is available, upon publication, at: https://doi.org/10.1037/tam0000168

coherent than this one, which could suggest that the person is either under the influence of drugs here and less here, or something."

"I see some linguistic links, though I am not a linguistic expert. He uses a lot of the same words. So, my impression is this is the same writer, threatener."

"I'm looking at kind of the graphic nature of the note. One of the things you would see is use of the... whenever he uses the letter ' $g$ ', you will see a tail on the ' $g$ ' moving from right to left in both letters. And it's very... also with the 'y'"s too. And it's very consistent throughout the note."

When discussing first thoughts, several participants already mentioned they deemed the risk of violence to be low $(84.62 \%)$. Several different possible motivations were mentioned when discussing first thoughts $(69.23 \%)$, such as a financial motive or hoping to create fear. Participants also made remarks about possible indicators for a mental health disorder $(61.54 \%)$. Almost half of the participants made remarks about wanting to seek further information $(46.15 \%)$.

"I would ask around as well to see if other people have any letters of this individual and whether they have been acted upon."

"I would again wanna check delivery method, postage, was it put in an envelope, where are the postage stamps."

Security measures and investigation

When asked which security measures should be put in place in response to the threats, several different approaches emerged from the responses relating to both security measures and possible investigative steps to be taken. They are shown in Table 2.

Table 2

Security and investigative themes

\begin{tabular}{lll}
\hline Theme & \% & $\boldsymbol{n}$ \\
\hline Look out for suspicious behavior & 69.23 & 9 \\
Start (police) investigation & 46.15 & 6 \\
Encourage alertness & 38.46 & 5 \\
Find out about persons with grudges & 30.77 & 4 \\
Do nothing & 30.77 & 4 \\
Check CCTV & 23.08 & 3 \\
Find out about other letters & 23.08 & 3 \\
Find out about other incidents & 23.08 & 3 \\
Forensic investigation (physical evidence) & 15.38 & 2
\end{tabular}


CAmerican Psychological Association, 2022. This paper is not the copy of record and may not exactly replicate the authoritative document published in the APA journal. The final article is available, upon publication, at: https://doi.org/10.1037/tam0000168

\begin{tabular}{lll}
\hline Look out for product tampering & 15.38 & 2 \\
Deal with anxiety of target & 7.69 & 1 \\
Install (physical) security & 7.69 & 1 \\
Consult product tampering expert & 7.69 & 1 \\
Meet for money transfer & 7.69 & 1 \\
Wait for more letters & 7.69 & 1 \\
\hline
\end{tabular}

\section{Author characteristics}

Two letters were presented to interviewees and no information was given regarding the relation between the two. The majority of participants expected the two letters to have been written by the same author(s) $(53.85 \%)$. The majority also expected there to be a single person, rather than a group, behind the threats $(84.62 \%)$. Most interviewees expected the author to be male $(69.23 \%)$, but several also mentioned they didn't know for sure $(46.15 \%)$ :

"In general from a profiling point of view I'm very careful about characteristics."

"I probably wouldn't. I'm not a big fan of profiling. I think it's mostly (...), there's a lot of guess in there. I need to know a lot more that is pattern behavior."

With regards to the relation between the author and target, several interviewees noted the possibility of the author living in the neighborhood $(46.15 \%)$, some suspected they were a former employee $(30.77 \%)$ or frequent visitor $(23.08 \%)$ of the target. Three interviewees inferred there was no relation between the author and target $(23.08 \%)$.

\section{Motivation}

Some participants already mentioned possible motivations when asked about their first thoughts $(69.23 \%)$, but interviewees were also asked to specifically state what they thought the author's intention was behind sending the ACT. Several possible motivations for sending the threats were mentioned, with the majority expecting it to be financial $(53.85 \%)$. Other possibilities included creating fear $(46.15 \%)$, harboring a grudge $(46.15 \%)$, resulting from mental illness (38.46\%), or seeking attention (30.77\%).

\section{Personality and mental health}

Regarding the possible personality and mental health of the threat author, a large variety of possibilities was raised in the interviews. Delusions were a common theme in responses to questions about both personality and mental health. Although some participants did not want to make any claims about these issues, several possible diagnoses were mentioned by others, such as Autism Spectrum Disorder (ASD), psychopathy, and psychosis. They are depicted in Table 3. Please note that participants were asked about personality and mental health separately, but certain themes appeared in response to both questions (e.g., delusions, narcissism) 
CAmerican Psychological Association, 2022. This paper is not the copy of record and may not exactly replicate the authoritative document published in the APA journal. The final article is available, upon publication, at: https://doi.org/10.1037/tam0000168

Table 3

Personality and mental health themes mentioned

\begin{tabular}{llllll}
\hline Personality & \multicolumn{5}{c}{ Mental health } \\
\hline Theme & $\%$ & $\boldsymbol{n}$ & Theme & $\%$ & $\boldsymbol{n}$ \\
Don't want to say & 38.46 & 5 & Delusions & 53.85 & 7 \\
Delusional & 38.46 & 5 & Disordered (unspecified) & 30.77 & 4 \\
Inadequate & 23.08 & 3 & Psychotic & 30.77 & 4 \\
Unstructured & 23.08 & 3 & Paranoia & 15.38 & 2 \\
Rational & 15.38 & 2 & ASD & 15.38 & 2 \\
Antisocial & 15.38 & 2 & No disorder & 15.38 & 2 \\
Low intelligence & 15.38 & 2 & Don't want to say (specific) & 15.38 & 2 \\
Sadist & 15.38 & 2 & Personality disorder & 7.69 & 1 \\
Don't know & 7.69 & 1 & Histrionic personality disorder & 7.69 & 1 \\
Frustrated & 7.69 & 1 & Borderline & 7.69 & 1 \\
Narcissistic & 7.69 & 1 & Psychopathy & 7.69 & 1 \\
Lack of empathy & 7.69 & 1 & Narcissistic personality disorder & 7.69 & 1 \\
& & & Grandiose delusions & 7.69 & 1 \\
\hline
\end{tabular}

Risk level

Twelve out of thirteen interviewees expected the author to send similar letters to other targets $(92.31 \%)$. Nevertheless, the majority of interviewees stated the risk level of the case was low $(76.92 \%)$. One interviewee said it was moderate $(7.69 \%)$, whereas two said it was not possible to determine $(15.38 \%)$.

External expertise

Participants were also asked what kind of external expertise they would involve, if any, to assess the case. Several participants said they would consult a handwriting expert $(53.85 \%)$, a linguist $(46.15 \%)$ or the police or private investigator $(46.15 \%)$. Some interviewees would wish to discuss the case with other threat assessment professionals $(23.08 \%)$ :

"I'll utilize colleagues to kind of do that double checking, triple checking. So, this has occurred today much faster than I would normally spend. So, it's that peer review that should be done, it should never be done by one person."

"And at least I'd say, at least two experts who have some experience with threat assessment to have a look at the letter, just also to put in discussion between persons." 
CAmerican Psychological Association, 2022. This paper is not the copy of record and may not exactly replicate the authoritative document published in the APA journal. The final article is available, upon publication, at: https://doi.org/10.1037/tam0000168

\section{Confidence in assessment}

When asked about their confidence level for the case they just assessed, the majority of participants noted they were confident (61.54\%), moderately confident $(23.08 \%)$, or that they had low confidence $(15.38 \%)$ :

"Absolute 100\% confidence in saying here's what I can tell you based on the letter, here [are the] investigative leads we can run, here's my assessment, here are some management strategies. I have full confidence in doing that because I do it every day."

"Well, at this stage I have to say everything is a hypothesis. So, it's not about me saying this is my judgment, goodbye. And that's a $60 \%$ confidence."

"Given that it's just a couple of letters, not a great deal [of confidence]. Not a great deal, reasonably low."

"[I have] fair confidence. Unless there's something unexpected that comes out in an interview or something like that I would say fair confidence."

"80\% [confidence], I'm fairly sure about this."

\section{Discussion}

The interviews revealed several important points about the assessment of anonymous threatening communications. In this section, we discuss five observations drawn from the results, concerning 1) inconsistencies in advice and assessment, 2) factors not pursued, 3) lack of practical or justifiable advice, 4) teamwork and external experts, 5) confidence, 6) negative opinions about offender profiling, and 7) lack of structure. Following this, aforementioned points are summarized and translated into eight recommendations for the future of assessing ATCs.

\section{(In) consistencies in advice and assessment}

The majority of interviewees in the study stated that the risk of violence in the case they assessed was low. All in all, this seems to be the sole point on which most interviewees agree. Because the suspect in the real-life case was caught before they could actualize their threat, it is difficult to ascertain to what extent participants were correct in their judgment of risk. It is important to note that it was not the aim of this study to test the extent to which expert threat assessors accurately estimate the risk of violence. Instead, the main concern of this study is the process by which practitioners arrive at these assessments in the case of an ATC. That is, in our view the consistency in risk assessments and the inconsistencies on other points is of more interest.

Importantly, the advice on how to manage the case varied widely between experts. Many experts came up with modest advice in which the victim was told to merely be alert and look out for suspicious behavior, without being specific about what suspicious behavior in fact involves. It was also suggested that the victim should do just nothing at all and simply wait. This was perhaps surprising considering it is not only the level of violence risk that should be a matter of concern and a task for threat assessment professionals, but also aspects such as 
managing victim's fear and the disruption that such threats cause for individuals or organizations involved. In contrast to these more muted responses, one interviewee went as far as suggesting a meeting be arranged with the possible suspect in order that law enforcement could detain them. Although an estimation of the level of risk is important, it is of greater importance that the subsequent risk management advice matches the assessment. Moreover, an equal amount of consistency in management advice should be expected as was observed in risk assessment judgments.

Besides the differences observed in risk management advice, further incongruence emerged in the assessment of specific characteristics of the suspect and the case. Several interviewees suggested gathering further information to enrich their understanding of the case (a key aspect of structured professional judgment). However, the factors requiring further information varied widely across interviewees. There was also a wide spectrum of possible explanations as to how the suspect may be related to the target, with some interviewees suggesting there was no relation at all (i.e., the communication was sent at random), and several others proposing the suspect was previously employed by the target organization. Although most interviewees agreed that the letters were authored by a single individual who was likely male, their assessment of the suspect's personality and mental health also provided grounds for disagreement. Several interviewees agreed the threatener must suffer delusions (both when asked about possible personality characteristics and mental health status), but an equal number of interviewees declined to speak on the suspect's personality. When asked directly about mental health, almost all interviewees were happy to offer a possible diagnosis, such as psychotic disorder or autism spectrum disorder. Just two out of thirteen interviewees declined to speak on mental health based on the letters alone.

It is possible that responses regarding the profile of the threat author are the result of illusory correlations, where assessors for example identified relationships between disorganized language and mental health issues, or between violent threats and the author's gender. These beliefs will most likely be (partially) determined by previous case experience of the threat assessor. Different experts will have different experiences and beliefs, resulting in different assessments. All in all, these results underline that there are difficulties associated with assessing an anonymous case based on communications alone. Highly trained and very experienced threat assessment practitioners gave strongly divergent assessments, management strategies, and profiles of the author of the communications. This raises the question to what extent the outcomes of cases and the concrete advice clients receive are likely to be determined by the threat manager who is enlisted for a case. Real life outcomes are likely to vary substantially because threat assessor inputs are so different, particularly in terms of enacting management strategies.

\section{Factors not pursued}

Participants assessed the case through semi-structured interview questions, and their observations will have undoubtedly been (partially) led by the questions that were asked. However, interviewees were encouraged on several occasions to offer their own thoughts, unguided by any prompt. Nevertheless, there were several factors that are commonly pursued in threat assessment but were not mentioned by any interviewee or only raised by a select few.

First, when asked about their first thoughts on the case, very few interviewees posed any questions about the target of the threat letters. In threat assessment for a known case, the assessor would gather evidence on the victim, for instance about their behavior in general, their position in the organization, personal and professional relationships, and any relevant incidents in the past (Meloy \& Hoffmann, 2014). Although approximately half of the participants did 
(C)American Psychological Association, 2022. This paper is not the copy of record and may not exactly replicate the authoritative document published in the APA journal. The final article is available, upon publication, at: https://doi.org/10.1037/tam0000168

report a wish to seek further contextual information, these largely focused on the method of delivery or other possible communications sent to other targets. These lines of inquiry are indeed recommended in previous work on ATC assessment procedures (Simons \& Tunkel, 2013, 2021)

Second, although offender motivation is such a crucial aspect when analyzing or assessing (criminal) behavior, most interviewees did not deeply explore the author's motivation in writing the threatening letters. Motivation and looking for leads in the letters that can help formulate hypotheses seem essential in this work. When asked, many interviewees presented back what the author was saying in his letters, thereby offering a superficial interpretation of motive rather than a more considered evaluation of the intent behind the act. The majority of participants also delved straight into language use and content of the letter when asked what their first thoughts were. Additionally, none of the participants discussed the possibility that the author of the communications was concealing true motivation or engaging in 'linguistic staging' (i.e., incorporating linguistic cues such as misspellings or disorganized language to mislead the reader), even though this possibility has been raised in previous literature on ATCs (Simons \& Tunkel, 2013).

One possible explanation for the lack of consideration for these and other factors is the problem of confirmation bias, anchoring heuristics, and selective perception. If participants were overly affected by an early hypothesis about the case, they may not have considered or pursued alternative cues or explanations. Access to a Structural Professional Judgment instrument, scientific literature, or professional discussions with colleagues may encourage assessors to take aforementioned (and other) factors into account and may help reduce the influence of bias.

\section{Lack of practical or justifiable advice}

Many interviewees presented their thoughts, ideas and psychological reflections about the cases, but very few translated these into practical advice for the investigator or victim. It was made explicit at the beginning of the interview that the expert had been approached by the head of security who was in need of practical guidance and advice. However, the focus of most responses to the questions posed failed to offer any constructive advice that could be adopted by the victimized organization's security manager.

A large proportion of respondents similarly failed to offer robust rationale for the observations they made. There was a tendency to propose characteristics about the offender, for example, without an accompanying justification for how and why these inferences were made and how this information could be subsequently used in management of the threat. Although it has not been assessed in this study, it is equally important to recognize that bias will also be present in the recipient of the threat assessment advice. Therefore, the potential for the advice offered by the practitioner to be misunderstood and therefore applied incorrectly by the recipient is significant. Consequently, the need to ensure that advice is unambiguous and not open to (mis)interpretation is vital. If we are advocating the use of our expertise in the assessment and management of anonymous threatening communications, it is crucial that the advice we provide is tangible, pragmatic and rationalized.

\section{Teamwork and external experts}

When asked about the approach to assessing ATCs, a large number of interviewees stressed the importance of working with others. While some noted they would seek out the opinion of other threat assessment experts on a case, others stated that teamwork was incorporated into their organization. For example, some interviewees were part of a threat assessment team that 
may consist of psychologists, psychiatrists, and/or investigators. Indeed, taking note of different viewpoints may guard against individual biases, such as selective perception. On the other hand, the risk of 'groupthink' is increased in a team setting, where striving for unanimity can become more important than critical evaluation (Janis, 1991). In the current study, participants were not able to converse with other experts even if they would usually do this in their work. Therefore, future work will also need to examine group processes in the assessment of ATCs and determine the consistency in assessments across different teams.

Several interviewees did suggest enlisting the help of expertise from other fields. Handwriting experts (i.e., graphologists) were most commonly mentioned. It is important to note that the validity of graphology has been called into question on several occasions in the last few decades and, within the scientific community, is largely seen as a debunked practice (e.g., Dazzi \& Pedrabissi, 2009; King \& Koehler, 2000; Neter \& Ben-Shakhar, 1989).

Others thought a forensic linguist may offer insight into the psychological and demographic profile of the suspect. The validity of author profiling through linguistic analysis in the context of threat assessment has recently been called into question (van der Vegt, Kleinberg, et al., 2020). Although there is evidence for relationships between language and gender (Newman et al., 2008), age (Pennebaker \& Stone, 2003), and personality (Pennebaker \& King, 1999), the effects are generally small and prediction performance is sub-optimal for use in practice (van der Vegt, Kleinberg, et al., 2020). In addition, the use of automated linguistic software was also mentioned by some interviewees. This software is generally aimed at providing a profile of a text author, derived from comparisons to texts written by terrorists and extremists (Akrami et al., 2018) or public figure threateners (Smith et al., 2013). It is important to note that such software needs to be adequately tested before use, and that current state-of-the art systems are not yet fit for practice (van der Vegt, Kleinberg, et al., 2020; van der Vegt, Mozes, et al., 2020).

It is relevant to note that there was a tendency amongst the interviewees to not be entirely clear about the boundaries of their own expertise. Whilst recommendations to involve graphologists or forensic linguists were often made, the interviewees were also prone to simultaneously making their own judgments about the handwriting or linguistic style of the author. There did not appear to be a reflection on when to say, 'I don't know, this is not my expertise' or 'I recommend you ask professional $\mathrm{x}, \mathrm{y}$ or $\mathrm{z}$ '.

\section{Confidence}

Interviewees offered many hypotheses regarding the case, but also often caveated their statements by saying they were not confident in some statements they made. As previously highlighted, whilst professing a lack of expertise in some aspects of the case information (e.g., handwriting) or the inappropriateness of drawing inferences about the offender, most participants were still willing to offer their opinions on these matters. This may be an artefact of the study design in which participants felt they could speculate more than in a real-life, highstake case. Nevertheless, it appears that interviewees were willing to draw very strong conclusions (e.g., on mental health diagnosis, gender and risk of violence) based on only two letters from an unknown source and with very little contextual information. That is, several participants ran to conclusions with very little objective support.

The interpretation of confidence in assessing the specific case and ATCs in general varied widely. Some participants stated they were confident in relaying their hypotheses based on what they could see in the communications, whereas others noted that they were not confident in their assessment based on the communications alone. Some noted that they would have been more confident if they were able to work in a team. Although there is not enough 
(C)American Psychological Association, 2022. This paper is not the copy of record and may not exactly replicate the authoritative document published in the APA journal. The final article is available, upon publication, at: https://doi.org/10.1037/tam0000168

data in the current study to examine this, future research may focus on the effect of the characteristics of a case (e.g., amount of contextual information available), and the assessors themselves (e.g., gender, cultural background) on confidence levels.

\section{Negative opinions about offender profiling}

It was interesting to find that a couple of (negative) comparisons to 'profiling' practices were made by interviewees. In fact, the precedent for applying a structured, empirically-based approach to the assessment of an unknown perpetrator can perhaps be found within this field, which has developed into what is now known as Behavioral Investigative Advice (BIA). It is possible that negative opinions expressed in the interviews stem from a lack of appreciation for how the discipline has evolved in recent years. Whilst its nascent application was within the Behavioral Science Unit of the FBI, it is only within this century that it has been transformed across the UK and a number of European countries into an established profession in its own right (Davis et al., 2018). Behavioral investigative advice, often referred to interchangeably (although unhelpfully) as offender profiling or investigative psychology, is concerned with the objective evaluation of the actions of an unknown perpetrator. By evaluating aspects of an offender's behavior, e.g., how an offender interacts with their environment and chosen target, choice of control methods, levels of violence exhibited, the manner in which they leave the scene, etc., it is possible for the practitioner to make more accurate inferences about how and why an offender has behaved in that way. Behavioral investigative advice offers an array of services to an investigation such as investigative hypotheses prioritization, nominal prioritization, (i.e., suggesting which potential suspects an investigation should focus their resources on first), and suspect interview strategy; it is by no means limited to the prediction of offender characteristics as it is often portrayed (Rainbow \& Gregory, 2011).

For the most part, Behavioral Investigative Advisers (BIAs) are called upon by law enforcement to assist with developing lines of enquiry to identify potential suspects in interpersonal, serious and sexual offences, based upon an offender's actions at a crime scene. Contemporary BIA practice draws upon the expertise of the practitioner in identifying the relevance of behavior exhibited by an offender, but its foundations are laid within the academic literature base in order to ensure that any inferences offered are robust, constructive and meaningful to investigative decision-making. For example, the prediction of an offender's characteristics for the purposes of suspect generation/prioritization will be purposefully limited to those that can be actioned by police investigations (i.e., relationship with victim, age, criminal history, etc.) and supported with empirical references. Reports may include such arguments as: "It is highly likely that the offender will have previously come to the attention of police for offences of violence. $82 \%$ of offenders on the ViCLAS database ${ }^{4}$ who exhibit behaviors $\mathrm{A}$ and $\mathrm{B}(n=672)$ have previous convictions for assault and/or homicide, and research by $\mathrm{X}$ (date) highlights the close correlation between these behaviors and hostile interactions" ${ }^{5 "}$. As this example illustrates, by deconstructing inferences into constituent parts as highlighted in Toulmin's (1958) philosophy of argument, such as the 'claim' (the offender will have come to the attention of police for violence), the 'strength of the claim' ( $82 \%$ of similar offenders had a violent conviction), the 'backing' (research by X), etc., there is a clarity

\footnotetext{
${ }^{4}$ Violent Crime Linkage Analysis System: https:/www.nationalcrimeagency.gov.uk/what-we-do/how-wework/providing-specialist-capabilities-for-law-enforcement/serious-crime-analysis

${ }^{5}$ This is an example of what an excerpt from a report within the context of BIA may look like. It is not an actual quote, and has been included for illustrative purposes.
} 
and transparency to the BIA's presentation of inferences which maximizes the likelihood that they will be communicated effectively (Alison et al., 2003).

Importantly, BIAs are increasingly being requested to support investigations involving anonymous threat letters; the communication is, after all, the result of an action by an unknown offender. BIAs are able to apply their blend of psychological insight and investigative experience to the analysis of a threatening or malicious communication, thereby ensuring that any inferences they make about the unknown offender or his/her actions are translated into a pragmatic outcome for the investigating officers. Just as within an interpersonal offence, they will consider the context within which the offence occurs, the victimology and any potentially linked incidents, as well as the contents of the letter itself. Therefore, BIAs may have much to offer the field of anonymous threat assessment, for instance as members of multi-disciplinary teams.

\section{Lack of structure}

Finally, and perhaps most fundamentally, the interviews provided some additional support that there is a lack of standardized protocol and Structured Professional Judgment tools in the case of anonymous threatening communications. This becomes clear based on the wide variance in approaches to the case and the variety in assessments made on several different factors. Furthermore, when assessors were asked about their first thoughts on the case or possible characteristics of the author, there was no single theme that united all participants.

Without a validated tool and limited empirical research on ATCs, experts make assessments based on intuition and experience, resulting in strongly divergent responses. A lack of structure in the approach to ATCs may also exacerbate the problem of selective perception and confirmation bias, where experts interpret information in light of experience on a previous case. In the presence of a SPJ tool, practitioners may be urged to confront these biases or will be reminded of alternative explanations and factors to consider.

\section{Limitations}

There are important limitations to be noted with regards to this study. Most importantly, the circumstances in which the experts performed their threat assessment will have been markedly different from their day-to-day practice. As has been raised in the discussion, participants who normally work in a team did not have the ability to do so in this study, while others may normally take more time for their assessment or have access to particular tools and software. Furthermore, it is a likely possibility that several claims made by interviewees in the study will not have been made if they were assessing the same case in real-life. The setup of this study will undoubtedly have allowed participants to speculate more, and thus we do not wish to claim that participants assess their real-life cases in precisely the same way. Nevertheless, we have sought to minimize the effect of the 'artificial' setup of this study on the interview responses and outcome of analyses. For example, participants received a lot of freedom when assessing the case (e.g., possibility to take notes) and were asked on several occasions which procedures and tools they would apply in a 'normal' situation. Furthermore, participants were never encouraged to make claims they were uncertain about and had ample opportunities to caveat their statements or refrain from responding to a question. Indeed, several participants did choose to do so, for example in response to questions about the author's personality and mental health. All in all, it is important to appreciate both the possible limitations to the generalizability of this study, as well as the efforts made to minimize the effect of the study design on the results. 
Another important limitation concerns the sample size of this study in addition to the fact that participants were obtained through convenience sampling. Therefore, we are not able to draw any statistical conclusions (e.g., on the relationship between years of experience and confidence in assessments) or widely generalizable conclusions. However, we hope the current findings lay the groundwork for future quantitative study of ATC assessment. For instance, the current design could be performed with a larger sample and by means of a survey in which questions can be asked on a scale (e.g., 'On a scale from 1-10, how confident are you about your assessment?). With a larger sample of experts, it will also be interesting to assess general accuracy in judgments of violence risk in the case of an anonymous written threat. Researchers could also compare a sample of experienced threat assessors who receive formal training in threat assessment versus a comparison group which does not, in order to assess the possible effect of such formal training on accuracy, for example. In order to judge assessment accuracy, it is important to select a case for which the actual outcome is known.

\section{Thoughts for consideration}

All in all, there was agreement between participants on the risk of violence in the case assessed. Together, the interviewed experts raised important factors for assessing ATCs that covered a broad range of subjects. Unfortunately, this is also problematic considering the assessment and advice concerning ATCs seems to be highly dependent on the expert him/herself. In light of these results and our own experience within the field of threat assessment, we have formulated some thoughts for consideration for the future development of ATC assessment:

1. Threat assessment practitioners should ensure that their risk management advice aligns with their assessment of violence risk.

Each case is unique and requires its own risk management strategy. However, if one judges the risk of violence to be low, strategies which require a disproportionate application of resources or effort (e.g., physical forensics, suspect surveillance) should be avoided, and vice versa.

2. Threat management advice should be practical and rationalized.

Study participants often made general statements (e.g., 'remain cautious'), which were not always supported with an underlying rationale. In order to adequately assist clients, risk management strategies should be as specific as possible (e.g., 'install CCTV at the entrance' or 'ask the Human Resources department to list incidents and conflicts they had with employees during the past 2 years'). The underlying rationale should also be explained.

3. Practitioners should allow for uncertainty, and adequately caveat their statements.

Strong statements based on limited evidence or concerning areas in which one is not an expert should be avoided. For instance, it is not advisable to draw strong conclusions from the language use in a communication if one does not have specific expertise in linguistic analysis. Moreover, if there is no empirical evidence for a link between specific (linguistic) cues and subsequent behavior or offender characteristics, definitive conclusions should not be drawn from such cues. Inconclusive judgments regarding the offender profile and violence risk are still preferred over strong, but wrong statements.

4. It is important to pursue contextual factors (beyond the communication) as much as possible.

Motivations for writing an ATC may be purposefully concealed by the author, thus relying on content alone is unreliable. Although this practice is widely accepted in the 
assessment of non-anonymous communications, victimology and contextual factors were frequently disregarded in the current study.

5. Future research should examine group processes and biases in the specific case of assessing ATCs.

Many practitioners work in a team, but little is known about how teams come to a joint assessment, and which processes and biases from the decision-making literature can be generalized to the specific case of group-based (anonymous) threat assessment.

6. Practitioners should be cautious in relying on external experts or tools, particularly if there is limited or contradictory evidence regarding their effectiveness.

Several experts stated they would consult expertise or rely on tools that have been debunked (graphology) or for which there is limited evidence regarding its validity (linguistic software). Furthermore, novel tools should be used to supplement one's assessment, not replace it.

7. Anonymous threat assessment may gain from the field of behavioral investigative advice.

BIA is similarly concerned with unknown perpetrators and has spent the last twenty year developing appropriate methods for the assessment of behavior and the delivery of robust and pragmatic advice. It may thus have much to offer to the field of anonymous threat assessment. Contrary to popular belief, it focuses on objective evaluation of the actions of an unknown perpetrator rather than a prediction of offender characteristics.

8. In light of the divergence in assessments in this study, a Structured Professional Judgment tool for assessing ATCs will be a worthwhile future endeavor. By using an SPJ, practitioners will be urged to consider alternative cues and hypotheses, thereby potentially reducing confirmation bias. It will likely also provide structure in procedure and stronger homogeneity in assessments, which was generally lacking in the current study of assessing ATCs.

\section{Conclusion}

This study examined assessment procedures in the case of anonymous threatening communications by interviewing threat assessment experts on their approach to the same case. Results showed that although experts largely agreed on their assessment of violence risk, they were highly divergent in their management advice and assessments of communication content and characteristics of the suspected author. By studying the nature and assessment procedures of ATCs, we can further structure threat assessment approaches and thereby improve our ability to prevent violence and promote safety. 
(C)American Psychological Association, 2022. This paper is not the copy of record and may not exactly replicate the authoritative document published in the APA journal. The final article is available, upon publication, at: https://doi.org/10.1037/tam0000168

\section{References}

Akrami, N., Shrestha, A., Berggren, M., Kaati, L., Obaidi, M., \& Cohen, K. (2018). Assessment of risk in written communication: Introducing the Profile Risk Assessment Tool (PRAT). EUROPOL. http://urn.kb.se/resolve?urn=urn:nbn:se:uu:diva-367346

Alison, L., Smith, M. D., Eastman, O., \& Rainbow, L. (2003). Toulmin's philosophy of argument and its relevance to offender profiling. Psychology, Crime \& Law, 9(2), 173-183. https://doi.org/10.1080/1068316031000116265

Borum, R., Fein, R., Vossekuil, B., \& Berglund, J. (1999). Threat assessment: Defining an approach to assessing risk for targeted violence. Behav. Sci. Law, 16.

Calhoun, F. S., \& Weston, S. W. (2017). Threat Assessment and Management Strategies: Identifying the Howlers and Hunters, Second Edition. CRC Press.

Chapman, L. J., \& Chapman, J. P. (1967). Genesis of popular but erroneous psychodiagnostic observations. Journal of Abnormal Psychology, 72(3), 193-204. https://doi.org/10.1037/h0024670

Davis, M., Rainbow, L., Fritzon, K., West, A., Brooks, N., Rainbow, L., Fritzon, K., West, A., \& Brooks, N. (2018, May 20). Behavioural investigative advice: A contemporary commentary on offender profiling activity. The Psychology of Criminal Investigation; Routledge. https://doi.org/10.4324/9781315637211-11

Dazzi, C., \& Pedrabissi, L. (2009). Graphology and Personality: An Empirical Study on Validity of Handwriting Analysis. Psychological Reports, 105(3_suppl), 1255-1268. https://doi.org/10.2466/PR0.105.F.1255-1268

Dietz, P. E., Matthews, D. B., Martell, D. A., Stewart, T. M., Hrouda, D. R., \& Warren, J. (1991). Threatening and Otherwise Inappropriate Letters to Members of the United States Congress. Journal of Forensic Sciences, 36(5), 13165J. https://doi.org/10.1520/JFS13165J

Dietz, P., Matthews, D. B., Duyne, C. V., Martell, D. A., Parry, C. D. H., Tracy, S., Warren, J., Crowder, J. D., \& Duyne, V. (1991). Threatening and Otherwise Inappropriate Letters to Hollywood Celebrities. Journal of Forensic Sciences, 36(1), 185-209.

Elbogen, E. B. (2002). The process of violence risk assessment: A review of descriptive research. Aggression and Violent Behavior, 7(6), 591-604. https://doi.org/10.1016/S1359-1789(01)00051-9

Geurts, R., Göteborgs universitet, \& Psykologiska institutionen. (2017). Interviewing to assess and manage threats of violence. Department of Psychology, University of Gothenburg.

Gigerenzer, G. (2008). Why Heuristics Work. Perspectives on Psychological Science, 3(1), 20-29. https://doi.org/10.1111/j.1745-6916.2008.00058.x

Goswami, S., Sarkar, S., \& Rustagi, M. (2009, March 20). Stylometric Analysis of Bloggers' Age and Gender. Third International AAAI Conference on Weblogs and Social Media. Third International AAAI Conference on Weblogs and Social Media. https://www.aaai.org/ocs/index.php/ICWSM/09/paper/view/208

Hart, S. D., Douglas, K. S., \& Guy, L. S. (2016). The Structured Professional Judgement Approach to Violence Risk Assessment. In The Wiley handbook on the theories, assessment and treatment of sexual offending (pp. 643-666).

https://onlinelibrary.wiley.com/doi/abs/10.1002/9781118574003.wattso030

Holmes, D. I. (1998). The Evolution of Stylometry in Humanities Scholarship. Literary and Linguistic Computing, 13(3), 111-117. https://doi.org/10.1093/1lc/13.3.111 
Iverson, G. L., Brooks, B. L., \& Holdnack, J. A. (2008). Misdiagnosis of cognitive impairment in forensic neuropsychology. Neuropsychology in the Courtroom: Expert Analysis of Reports and Testimony, 243-266.

James, D. V., MacKenzie, R., \& Farnham, F. R. (2014). Communications Threat Assessment Protocol. Theseus LLP.

Janis, I. (1991). Groupthink. In A First Look at Communication Theory. McGrawHill.

Keren, G., \& Teigen, K. H. (2004). Yet Another Look at the Heuristics and Biases Approach. In Blackwell Handbook of Judgment and Decision Making (pp. 89-109). John Wiley \& Sons, Ltd. https://doi.org/10.1002/9780470752937.ch5

King, R. N., \& Koehler, D. J. (2000). Illusory correlations in graphological inference. Journal of Experimental Psychology: Applied, 6(4), 336-348. https://doi.org/10.1037/1076-898X.6.4.336

Meloy, J. R. (2015). Threat assessment: Scholars, operators, our past, our future. Ournal of Threat Assessment and Management, 2(3-4), 231-242.

Meloy, J. R., \& Hoffmann, J. (2013). International Handbook of Threat Assessment. Oxford University Press, Incorporated. http://ebookcentral.proquest.com/lib/ucl/detail.action?docID=1573156

Monahan, J., Steadman, H. J., Silver, E., Appelbaum, P. S., Robbins, P. C., Mulvey, E. P., Roth, L. H., Grisso, T., \& Banks, S. (2001). Rethinking Risk Assessment: The MacArthur Study of Mental Disorder and Violence. Oxford University Press.

Murray, J., \& Thomson, Dr. M. E. (2010). Applying decision making theory to clinical judgements in violence risk assessment. Europe's Journal of Psychology, 6(2), 150 171. https://doi.org/10.5964/ejop.v6i2.189

Neter, E., \& Ben-Shakhar, G. (1989). The predictive validity of graphological inferences: A meta-analytic approach. Personality and Individual Differences, 10(7), 737-745. https://doi.org/10.1016/0191-8869(89)90120-7

Newman, M. L., Groom, C. J., Handelman, L. D., \& Pennebaker, J. W. (2008). Gender Differences in Language Use: An Analysis of 14,000 Text Samples. Discourse Processes, 45(3), 211-236. https://doi.org/10.1080/01638530802073712

Nickerson, R. S. (1998). Confirmation Bias: A Ubiquitous Phenomenon in Many Guises. https://journals.sagepub.com/doi/abs/10.1037/1089-2680.2.2.175

Open Science Framework (2021). Assessment procedures in anonymously written threats of harm and violence. Retrieved from: https://osf.io/5twzu/?view_only=0c7744e97bd74957ac30f06e44337c7b

Pennebaker, J. W. (2011a). Using computer analyses to identify language style and aggressive intent: The secret life of function words. Dynamics of Asymmetric Conflict, 4(2), 92-102. https://doi.org/10.1080/17467586.2011.627932

Pennebaker, J. W. (2011b). The secret life of pronouns. New Scientist, 211(2828), $42-45$. https://doi.org/10.1016/S0262-4079(11)62167-2

Pennebaker, J. W., \& King, L. A. (1999). Language Use as an Individual Difference. Journal of Personality and Social Psychology, 77(6), 1296-1312. https://doi.org/10.1037/0022-3514.77.6.1296

Pennebaker, J. W., \& Stone, L. D. (2003). Words of wisdom: Language use over the life span. Journal of Personality and Social Psychology, 85(2), 291-301. https://doi.org/10.1037/0022-3514.85.2.291

QSR Internation Pty Ltd. (2014). NViVo (Version 10). https://www.qsrinternational.com/nvivo-qualitative-data-analysis-software/home 
Rainbow, L., \& Gregory, A. (2011). What Behavioural Investigative Advisers actually do: Lee Rainbow and Adam Gregory. In Professionalizing Offender Profiling. Routledge.

Simons, A., \& Tunkel, R. (2013). The Assessment of Anonymous Threatening Communications. In International Handbook of Threat Assessment. Oxford University Press.

Simons, A., \& Tunkel, R. (2021). The Assessment of Anonymous Threatening Communications. In International Handbook of Threat Assessment (2nd Edition). Oxford University Press.

Smith, S. S., Woyach, R. B., \& O’toole, M. E. (2013). Threat Triage: Recognizing the Needle in the Haystack. In International Handbook of Threat Assessment.

Toulmin, S. (1958). The Uses of Argument. Cambridge University Press. https://www.cambridge.org/core/books/uses-ofargument/26CF801BC12004587B66778297D5567C

Tversky, A., \& Kahneman, D. (1974). Judgment under Uncertainty: Heuristics and Biases. Science, 185(4157), 1124-1131. https://doi.org/10.1126/science.185.4157.1124

van der Vegt, I., Kleinberg, B., \& Gill, P. (2020). Too Good to be True? Predicting Author Profiles for Abusive Language.

van der Vegt, I., Mozes, M., Kleinberg, B., \& Gill, P. (2020). The Grievance Dictionary: Understanding Threatening Language Use.

Wallace, P. (2015). The Psychology of the Internet. Cambridge University Press. 